\title{
Modelamiento y prognosis estadística y cartográfica del cambio en el uso de la tierra
}

\author{
Modelling and prognosis of land uses changes \\ VICTOR SANDOVAL ${ }^{1}$, PEDRO REAL ${ }^{2}$ \\ ${ }^{1}$ Facultad de Ciencias Forestales, Universidad Austral de Chile, Valdivia. E-mail: vsandova@uach.cl \\ ${ }^{2}$ Facultad de Ciencias Forestales, Universidad de Concepción, Concepción. E-mail: preal@udc.cl
}

\begin{abstract}
SUMMARY
The statistical-space modeling of change in the use of the ground is derived from the combined use of cartographic techniques, GIS, and multivariant statistical models. The primary objective is to identify the magnitude and space distribution of change and to cartographically project and unfold the probability of occurrences in the near future, under the assumption that the techniques of ground utilization will continue to be similar to those of the recent past. The more independent variables used in these types of models are: distance to markets, road infrastructure, topography, quality of ground, precipitation, and forest fragmentation. In the present article, the theoretical and practical aspects in space modeling of the earth are discussed.
\end{abstract}

Keywords: cartography, GIS, multivariant statistical models.

\section{RESUMEN}

La modelación estadístico-espacial del cambio en el uso del suelo, se deriva de la combinación y uso de técnicas cartográficas, sistemas de información geográfica y modelos estadísticos multivariantes. Su objetivo principal es identificar la magnitud y distribución espacial del cambio, proyectar y desplegar cartográficamente su probabilidad de ocurrencia en el futuro cercano, bajo el supuesto que las técnicas de manejo del suelo continuarán siendo similares al pasado cercano. Las variables independientes más utilizadas en este tipo de modelos son: distancia a los mercados, infraestructura vial, topografía, calidad de suelos, precipitación, fragmentación del bosque, etc. En el presente artículo se discuten aspectos teóricos y prácticos utilizados en el proceso de modelamiento espacial del uso de la tierra.

Palabras clave: cartografía, SIG, modelos estadísticos multivariantes.

\section{INTRODUCCION}

En las últimas dos décadas se han incrementado las investigaciones relacionadas con el análisis y modelamiento del cambio en el uso del suelo, principalmente los referidos al proceso de deforestación en países tropicales (1). Muchos de estos modelos están ligados a estudios del ciclo global del carbono o impulsados por la búsqueda de las causas y consecuencias del cam- bio climático, pérdida de biodiversidad o modelamiento del paisaje (2).

En general, los esfuerzos de analizar y modelar los procesos de cambio de uso del suelo se orientan a responder las siguientes interrogantes: ¿Por qué ocurre el cambio en el uso del suelo? ¿Dónde? y ¿cuándo ocurre el cambio? Estas tres interrogantes fundamentales son abordadas a través de metodologías apoyadas en el uso de sensores remotos y análisis estadístico espacial $(2,3)$. 
Una completa revisión de modelos relacionados al análisis del proceso de cambio en el uso del suelo, desde el punto de vista espacial, es descrita por Baker (4), Shugar et al. (5), Parks (6), Turner et al. (3), Elston et al. (7) y Lambin (8). En trabajos recientes Kaimowitz (1) efectúa una revisión de modelos económicos de deforestación, focalizándose principalmente en los que explican las causas y comportamiento de los propietarios de la tierra y la relación de sus decisiones con el resto de la economía. En esta interpretación socioeconómica del cambio del uso del suelo, específicamente de la deforestación tropical, el autor identifica causas, agentes de deforestación, parámetros de decisión e instrumentos políticos y económicos fundamentales que intervienen e interactúan en el proceso global de deforestación. Desde un punto de vista operacional Lambin (8) diferencia tres principales tipos de modelos para explicar el proceso de cambios en el uso del suelo: Empíricos, Algorítmicos y Sistémicos. Los primeros imitan las relaciones reales existentes entre las variables que explican el cambio de uso y asumen que estas continuarán así en el futuro. En los Modelos Algorítmicos los procesos individuales de un sistema son descritos por ecuaciones simples, pero bajo principios científicos. Finalmente, los modelos sistémicos se enfocan a explicar el funcionamiento e interacción de todos los componentes de un ecosistema.

Desde la perspectiva de los modelos estadístico-espaciales se destacan tres elementos importantes: (a) el vector de cambio que indica donde y cuando ocurre éste en el pasado y dónde ocurrirá en el futuro cercano, (b) la matriz o balance de superficies que representa la orientación del cambio, y (c) índices estadísticos de asociación entre áreas de cambio y los factores ambientales y socioeconómicos del área. Estos elementos, en el marco de una estrategia y flujo de modelamiento, se describen en la figura 1.

\section{MATERIAL Y METODOS}

Ejemplo numérico de observación, análisis y proyección espacial del cambio: Utilizando los componentes de análisis descritos, se estudia el proceso de transformación de los usos de la tierra, desde praderas, agrícolas y matorrales a plan- taciones forestales. El análisis está circunscrito a un período de 5 años, desde 1994 a 1998, sobre un área de 60.000 ha ubicadas en el valle central de la VIII Región.

La información alfanumérica y gráfica de la zona se estructuró en un modelo de datos relacional, considerando áreas con atributos ambientales y temáticos similares. Estas se denominan Unidades Homogéneas del Terreno (UHT) y se obtienen a partir de cruce digital entre las coberturas cartográficas disponibles y derivadas para este ejercicio.

Las UHT se describen según la siguiente expresión matricial:

$$
\mathrm{UHT}_{\mathrm{ij}}
$$

Donde:

$\mathrm{UHT}_{\mathrm{ij}}=$ Unidad Homogénea de terreno

$\mathrm{i} \quad=1,2 \ldots \ldots \mathrm{n}$

$\mathrm{j} \quad=1,2 \ldots \ldots \mathrm{k}$

$\mathrm{n} \quad=$ Número de unidades espaciales consideradas en el estudio

$\mathrm{k}=$ Número de variables o clases temáticas incluidas en el estudio

En el ejercicio las variables espaciales o categorías evaluadas en relación al cambio de uso son:

- Uso del suelo (inicio y término del período).

- Uso Potencial del suelo.

- Estructura y tamaño de propiedad.

- Distancia a caminos.

- Distancia a plantaciones forestales.

- Variables ambientales (pendiente, exposición, altitud).

Análisis de la dirección del cambio: La estructura de uso de la tierra para el año $1994\left(\mathrm{t}_{0}\right)$ y 1998 $\left(\mathrm{t}_{1}\right)$ del área de estudio se indican en el cuadro 2. Los datos señalan que el año 1994 el uso del suelo es representado principalmente por las clases agrícolas y plantaciones forestales con 35.200 ha $(57 \%)$ y 13.176 ha $(21,5 \%)$, respectivamente. En segundo orden de magnitud se encuentran las clases matorrales y praderas con el $7,7 \%$ y $7,4 \%$ de la superficie total. Las restantes clases de uso, cuerpos de agua, áreas urbanas, bosque natural y plantaciones jóvenes representan en total $2,1 \%$ de la superficie estudiada. 
1. Definir vector de cambio de uso del suelo:
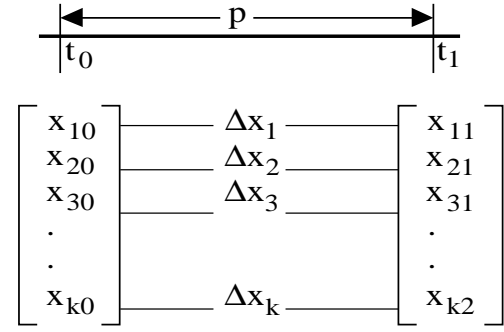

$\mathrm{X}_{\mathrm{k} 0}=$ Clase de uso del suelo $\mathrm{k}$ al año $\mathrm{t}_{0} \mathrm{o}$ inicio del período de estudio

$\mathrm{X}_{\mathrm{k} 1}=$ Clase de uso del suelo $\mathrm{k}$ al año $\mathrm{t}_{1} \mathrm{o}$ término del período de estudio

$\Delta \mathrm{x}_{\mathrm{k}}=$ Cambio absoluto del uso del suelo $\mathrm{k}$ en el período de tiempo $\mathrm{p}_{\mathrm{t}}=\mathrm{t}_{0}-\mathrm{t}_{1}$

$\mathrm{t}_{0}=$ Año de inicio del período de calibración del modelo

$\mathrm{t}_{1}=$ Año término del período de calibración del modelo

2. Determinar la dirección del cambio en el uso del suelo:

\begin{tabular}{|c|c|c|c|c|c|}
\hline \multirow{2}{*}{$\begin{array}{c}\text { Clases de } \\
\text { uso al } \\
\text { año }\left(\mathrm{t}_{0}\right)\end{array}$} & \multicolumn{4}{|c|}{$\begin{array}{l}\text { Clases de uso } \\
\text { al año }\left(\mathrm{t}_{1}\right)\end{array}$} & \multirow{2}{*}{$\begin{array}{l}\text { Superficie } \\
\text { total al } \\
\text { al año } t_{0}\end{array}$} \\
\hline & $\mathrm{x}_{11}$ & 21 & $\ldots$ & $\mathrm{x}_{\mathrm{k} 1}$ & \\
\hline $\mathrm{x}_{10}$ & $\mathrm{f}_{11}$ & $\Delta_{12}$ & $\ldots$ & $\Delta_{1 \mathrm{k}}$ & $\mathrm{F}_{10}$ \\
\hline $\mathrm{x}_{20}$ & $\Delta_{21}$ & $\mathrm{f}_{11}$ & $\ldots$ & $\Delta_{2 \mathrm{k}}$ & $\mathrm{F}_{10}$ \\
\hline$\cdots$ & $\ldots$ & $\ldots$ & $\ldots$ & $\ldots$ & $\cdots$ \\
\hline $\mathrm{x}_{\mathrm{k} 0}$ & $\Delta_{\mathrm{k} 1}$ & $\Delta_{\mathrm{k} 2}$ & $\ldots$ & $\mathrm{f}_{\mathrm{kk}}$ & $\mathrm{F}_{\mathrm{k} 0}$ \\
\hline $\begin{array}{l}\text { Superficie } \\
\text { total al año } t_{1}\end{array}$ & $\mathrm{~F}_{11}$ & $\mathrm{~F}_{21}$ & $\ldots$ & $\mathrm{F}_{\mathrm{k} 1}$ & $\mathrm{~F}$ \\
\hline
\end{tabular}

$\mathrm{x}_{\mathrm{k} 0}=$ Clase de uso del suelo $\mathrm{k}$ al año $\mathrm{t}_{0} \mathrm{o}$ inicio del período de estudio

$\mathrm{x}_{\mathrm{k} 1}=$ Clase de uso del suelo $\mathrm{k}$ al año $\mathrm{t}_{1} \mathrm{o}$ término del período de estudio

$\mathrm{f}_{\mathrm{kk}}=$ Superficie de la clase de uso $\mathrm{k}$ al año $\mathrm{t}_{0}$, que al año $\mathrm{t}_{1}$ permanece en la misma clase $\mathrm{k}$

$\mathrm{F}_{\mathrm{ko}}=$ Superficie total de la clase de uso $\mathrm{k}$ al año $\mathrm{t}_{0}$

$\mathrm{F}_{\mathrm{k} 1}=$ Superficie total de la clase de uso $\mathrm{k}$ al año $t_{1}$

$\mathrm{F}=$ Superficie total del área

$\Delta_{1 \mathrm{k}}=$ Cambio de uso de la clase $\mathrm{x}_{10}$ a la clase de uso $\mathrm{x}_{1 \mathrm{k}}$

3. Ajuste del modelo de cambio de uso del suelo y proyección espacial

Análisis Estadístico Multivariable: Ej.: Regresión logística

$$
\pi=\frac{\left(\exp \beta_{0}+\beta_{1} \chi_{1}+\beta_{2} \chi_{2}+\ldots+\beta_{\mathrm{k}} \chi_{\mathrm{k}}\right)}{1+\exp \left(\beta_{0}+\beta_{1} \chi_{1}+\beta_{2} \chi_{2}+\ldots+\beta_{\mathrm{k}} \chi_{\mathrm{k}}\right)}
$$

Donde:

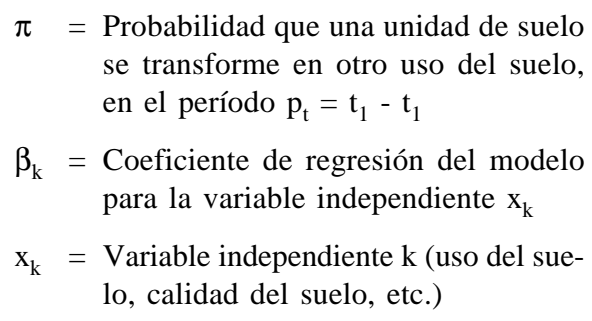

Figura 1. Principales componentes en una estrategia de observación, análisis y proyección espacial del cambio en el uso de la tierra.

Modules of the empirical, spatially-statistic model. 


\section{CUADRO 2}

Cambio absoluto de uso del suelo, periodo 1994-1998.

Land use changes in the period 1994-1998.

\begin{tabular}{|l|r|c|c|c|}
\hline Uso actual del suelo & $\begin{array}{c}\text { Superficie } \\
\text { (ha) }\end{array}$ & $\begin{array}{c}\text { Superficie 1998 } \\
\text { (ha) }\end{array}$ & $\begin{array}{c}\text { Diferencia } \\
\text { (ha) }\end{array}$ & $\begin{array}{c}\% \\
\text { (de 1994) }\end{array}$ \\
\hline Urbano & 75,9 & 75,9 & 0 & 0,0 \\
Agrícola & $35.200,5$ & $33.937,5$ & -1.263 & $-2,1$ \\
Praderas & $4.533,2$ & $2.824,1$ & $-1.709,1$ & $-2,8$ \\
Matorrales & $7.158,7$ & $7.709,8$ & 551,1 & 0,9 \\
Plantaciones (adultas) & $13.176,8$ & $9.265,0$ & $-3.911,8$ & $-6,4$ \\
Plantaciones (jóvenes) & 43,7 & $6.357,0$ & $6.313,3$ & 10,3 \\
Bosque Natural & 90,3 & 165,9 & 75,6 & 0,1 \\
Lagunas & $1.075,7$ & $1.019,8$ & $-55,9$ & $-0,1$ \\
Total & $61.354,8$ & $61.355,0$ & 0,2 & \\
\hline
\end{tabular}

Las modificaciones en el período $\mathrm{p}_{\mathrm{t}}$, según el cuadro 2 , se produjeron al interior de la clase de uso de plantaciones forestales, con un incremento de la sub-clase plantaciones jóvenes en 6.300 ha y una disminución de 3.955 ha en las plantaciones adultas, lo que corresponde $\mathrm{a}+10,3 \mathrm{y}-6,4 \%$ respectivamente. Por otra parte se observa una dismi- nución de la clase suelos agrícolas y ganaderos en 1.263 y 1.709 ha, lo que corresponde a un 2,1 y $2,6 \%$ de superficie total en 1994, respectivamente.

El balance de superficie que indica la matriz de cambio del uso del suelo (cuadro 3), posibilita la cuantificación del traspaso de superficie entre las diferentes clases de uso durante este período.

\section{CUADRO 3}

Matriz cruzada en el cambio de uso entre los años 1994 y 1998 (ha).

Cross table between land use classes of the years 1994 and 1998 .

\begin{tabular}{|l|r|r|r|r|r|r|r|r|r|}
\hline \multirow{2}{*}{$\begin{array}{c}\text { Uso del suelo } \\
\text { año 1994 }\end{array}$} & \multicolumn{9}{|c|}{ Uso del suelo año 1998 } \\
\cline { 2 - 9 } & Urbano & Agrícola & Praderas & Matorrales & $\begin{array}{c}\text { Plantaciones } \\
\text { adultas }\end{array}$ & $\begin{array}{c}\text { Plantaciones } \\
\text { jóvenes }\end{array}$ & $\begin{array}{c}\text { Bosque } \\
\text { nativo }\end{array}$ & $\begin{array}{c}\text { Lagunas } \\
\text { Total } \\
1994\end{array}$ \\
\hline Urbano & 76 & 0 & 0 & 0 & 0 & 0 & 0 & 0 & 76 \\
Agrícola & 0 & 33.938 & 0 & 901 & 152 & 210 & 0 & 0 & 35.201 \\
Praderas & 0 & 0 & 2.824 & 0 & 10 & 1.699 & 0 & 0 & 4.533 \\
Matorrales & 0 & 0 & 0 & 6.809 & 110 & 164 & 76 & 0 & 7.159 \\
Plantaciones adultas & 0 & 0 & 0 & 0 & 8.992 & 4.185 & 0 & 0 & 13.177 \\
Plantaciones jóvenes & 0 & 0 & 0 & 0 & 0 & 44 & 0 & 0 & 44 \\
Bosque nativo & 0 & 0 & 0 & 0 & 0 & 0 & 90 & 0 & 90 \\
Lagunas & 0 & 0 & 0 & 0 & 0 & 56 & 0 & 1.020 \\
Total 1998 & 76 & 33.938 & 2.824 & 7.710 & 9.265 & 6.357 & 166 & 1.020 \\
6
\end{tabular}


Las cifras indican que el incremento de superficies ocurrido en la clase de uso Plantaciones, subclase Plantaciones jóvenes, provienen principalmente de la subclase Plantaciones adultas con 4.185 ha $(65 \%$ de la superficie total de las clases al año 1998) y de la clase de uso Praderas que aporta 1699 ha $(27 \%)$. Las restantes superficies transferidas a plantaciones jóvenes provienen de los usos agrícolas y matorral con un total de 374 ha, 6\% de la superficie forestada al año 1998. Otro aspecto importante que se aprecia en la matriz de cambios es que el $95 \%$ de la superficie modificada en la clase de uso Praderas es transferido a plantaciones forestales. En forma similar se observa un flujo de superficies agrícolas a la clase de uso matorrales de 901 ha, lo que se interpreta como una primera fase de transferencia antes de ser transformada definitivamente a plantaciones forestales.

En la figura 3 se aprecia como los usos de la agricultura tradicional (cultivos agrícolas y praderas), como también la clase matorrales tienden a disminuir en favor de las plantaciones forestales. Se comprueba que la fuente de superficies para la forestación proviene principalmente entonces de las praderas y matorrales, y en menor medida de suelos agrícolas

Validación del modelo: Las relaciones espaciales inferidas anteriormente sobre el cambio de uso en las UHT se evaluaron en este ejercicio mediante la técnica estadística regresión-logística. Esta técnica ha sido utilizada principalmente para modelar el proceso de deforestación ocurrida en bosques tropicales (9). En el cuadro 4 se pre- sentan los resultados, en términos de relación espacial de cada variable (Correlación de Person) y su significancia estadística referida a la probabilidad de cambio de uso del suelo.

En el cuadro 4 se observa que las variables: tamaño de la propiedad, uso actual del suelo y uso potencial del suelo explican significativamente desde un punto de vista espacial, la ocurrencia de cambios en el área estudiada. Una menor significación estadística se observa para las variables exposición, distancia a caminos y plantaciones forestales establecidas.

Los valores entregados por esta técnica corroboran las tendencias de distribución espacial de las áreas de cambio observadas preliminarmente en la figura 2 y en la matriz de cambio (cuadro 3 ).

Con los análisis anteriores se pueden concluir que la existencia de las siguientes relaciones espaciales entre el proceso de cambio y las variables geofísicas son:

a) El cambio de uso del suelo en el área está ocurriendo principalmente en propiedades mayores a 300 ha. Esto se corrobora con la alta significación estadística de esta variable en el modelo LOGIT.

b) La categoría de uso potencial del suelo II está estrechamente correlacionada con la conversión del uso actual del suelo a plantaciones,

c) Las áreas clasificadas en el uso Praderas están siendo reemplazadas por plantaciones forestales. También se observa, aunque en menor medida, una tendencia de reemplazo hacia suelos agrícolas.

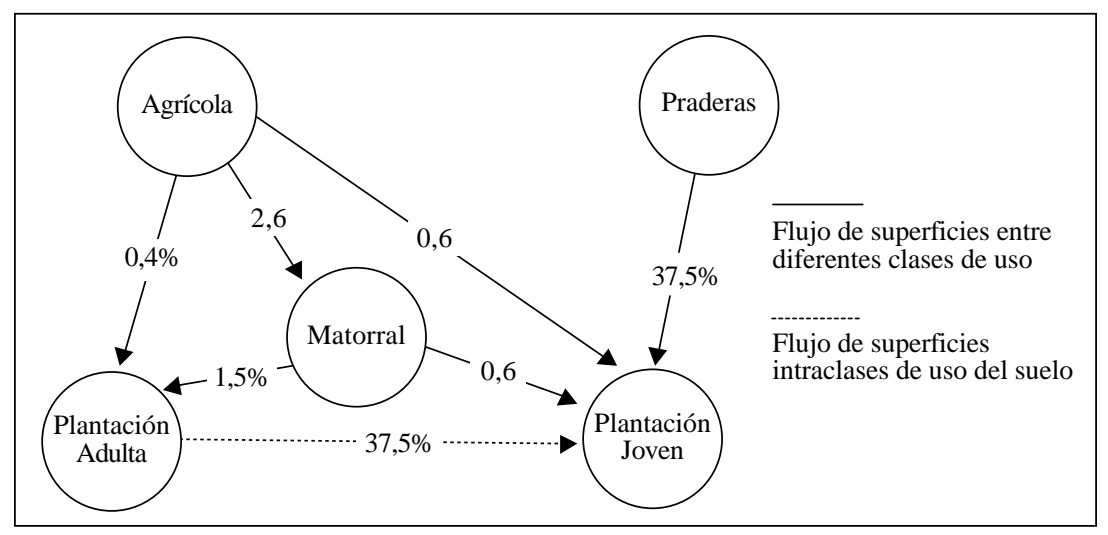

Figura 3. Modelo de cambio de uso actual del suelo en el área estudio.

Changes of land use in the investigation area. 


\section{CUADRO 4}

Modelo de Regresión logística y coeficientes de correlación de Person.

Model of the logistic regression.

\begin{tabular}{|c|c|c|c|c|}
\hline \multirow{2}{*}{$\begin{array}{l}\text { Factores que influyen } \\
\text { en el proceso de cambio }\end{array}$} & \multicolumn{4}{|c|}{ Parámetros de la regresión logística } \\
\hline & $\begin{array}{c}\text { Variables } \\
\text { Independientes }\end{array}$ & $\begin{array}{l}\text { Coefientes } \\
\text { del Modelo }\end{array}$ & $\begin{array}{l}\text { Wald Chi } \\
\text { Cuadrado }\end{array}$ & $\begin{array}{l}\text { Valores } \\
\text { de } \mathrm{Chi}^{2}\end{array}$ \\
\hline Uso actual del suelo & $\begin{array}{l}\text { Pradera } \\
\text { Agrícola } \\
\text { Matorrales }\end{array}$ & $\begin{array}{c}2,2402 \\
0,2515 \\
-\end{array}$ & $\begin{array}{c}576,72 \\
5,49 \\
-\end{array}$ & $\begin{array}{c}945,1 * * \\
5,51 \\
-\end{array}$ \\
\hline Uso potencial del suelo & $\begin{array}{l}\text { Clase I } \\
\text { Clase II } \\
\text { Clase> II }\end{array}$ & $\begin{array}{c}1,2492 \\
1,6474 \\
-\end{array}$ & $\begin{array}{c}18,7 \\
202,92 \\
-\end{array}$ & $\begin{array}{c}18,24 \\
290,3 * * \\
-\end{array}$ \\
\hline Tamaño de la propiedad & $\begin{array}{l}0-100 \text { ha } \\
100-300 \text { ha } \\
>300 \text { ha }\end{array}$ & $\begin{array}{c}- \\
0,5077 \\
1,1359\end{array}$ & $\begin{array}{c}- \\
14,28 \\
105,5\end{array}$ & $\begin{array}{c}- \\
16,96 \\
154,5 * *\end{array}$ \\
\hline Distancia a caminos & $\begin{array}{l}0-1.000 \mathrm{~m} \\
1.000-2.000 \mathrm{~m} \\
>2.000 \mathrm{~m}\end{array}$ & $\begin{array}{c}- \\
- \\
1,4864\end{array}$ & $\begin{array}{c}- \\
- \\
26,89\end{array}$ & $\begin{array}{c}- \\
- \\
50,94\end{array}$ \\
\hline Distancia a plantaciones & $\begin{array}{l}>1.000 \mathrm{~m} \\
1.000-2.000 \mathrm{~m} \\
>2.000 \mathrm{~m}\end{array}$ & $\begin{array}{c}-0,2054 \\
- \\
-0,9476\end{array}$ & $\begin{array}{c}6,02 \\
- \\
-29,35\end{array}$ & $\begin{array}{c}4,86 \\
- \\
32,5\end{array}$ \\
\hline Clases de Exposición & $\begin{array}{l}\mathrm{N} \\
\mathrm{E} \\
\mathrm{S} \\
\mathrm{O}\end{array}$ & $\begin{array}{l}2,1527 \\
2,6165 \\
2,87 \\
2,4531\end{array}$ & $\begin{array}{c}- \\
19,88 \\
24,09 \\
12,62\end{array}$ & $\begin{array}{r}- \\
16,59 \\
14,66 \\
6,99\end{array}$ \\
\hline Categorías de Pendiente & $\begin{array}{l}0-15 \% \\
15-30 \% \\
>30 \%\end{array}$ & $\begin{array}{c}- \\
-0,3113 \\
-\end{array}$ & $\begin{array}{c}- \\
10,41 \\
-\end{array}$ & $\begin{array}{c}- \\
11,083 \\
-\end{array}$ \\
\hline
\end{tabular}

**Significativos al $95 \%$.

Cálculo de la Matriz de probabilidad de cambio de las UHT: La probabilidad de cambio representa la susceptibilidad que tiene una determinada clase de uso del suelo en transformarse a otro estado o clase de uso diferente. Esta probabilidad se determinó para este ejercicio en función de las variables topográficas y temáticas más relevantes seleccionadas en el punto anterior. La probabilidad $\left(\pi_{\mathrm{i}}\right)$ se estimó a nivel UHT y propiedad privada. En ambos casos la probabilidad o susceptibilidad espacial que una UHT se transforme en plantación forestal, es función de los atributos que la componen, es decir, el tamaño de propiedad, la capacidad de uso potencial y el uso actual del suelo, lo que corresponde a la siguiente expresión:

$$
\pi_{\mathrm{UHTij}}=\mathrm{q}_{\mathrm{ij}},
$$

Donde:

$\pi_{\mathrm{UHTij}}$ : Probabilidad $\pi$ de cambio de uso del suelo de la unidad homogénea i de terreno;

$\mathrm{q}_{\mathrm{ij}:}$ Tasa de cambio real para una superficie caracterizada por los atributos temáticos k de la UHTi.

Para una propiedad privada específica, desde el punto de vista espacial, la probabilidad de cambio es $\pi_{\mathrm{UHTij}}$, ponderada por las distintas unidades homogénea UHTij que la conformen. 


\section{CUADRO 5}

Probabilidad de cambio a nivel de propiedad privada

Probabilities of changes

\begin{tabular}{|c|c|c|c|c|c|c|c|c|c|c|c|c|}
\hline \multirow[t]{3}{*}{ ID } & \multirow{3}{*}{$\begin{array}{c}\text { Superficie } \\
\text { de la parcela } \\
\text { o propiedad } \\
\text { ha }\end{array}$} & \multirow{3}{*}{$\begin{array}{c}\text { Superficie } \\
\text { forestable } \\
\text { al año } 1998 \\
\text { ha* }\end{array}$} & \multicolumn{3}{|c|}{ Agrícola } & \multicolumn{3}{|c|}{ Praderas } & \multicolumn{3}{|c|}{ Matorrales } & \multirow{3}{*}{$\begin{array}{c}\text { Probabilidad } \\
\% \text { de cambio } \\
\text { asociada a } \\
\text { cada propiedad }\end{array}$} \\
\hline & & & \multicolumn{9}{|c|}{ Capacidad de uso potencial del suelo } & \\
\hline & & & 1 & 2 & $>2$ & 1 & 2 & $>2$ & 1 & 2 & $>2$ & \\
\hline 1 & 12,2 & 12,2 & 0,0 & 4,9 & 0,0 & 0,0 & 0,0 & 0,0 & 0,0 & 7,3 & 0,0 & 0,4 \\
\hline 2 & 12,3 & 2,8 & 1,4 & 1,4 & 0,0 & 0,0 & 0,0 & 0,0 & 0,0 & 0,0 & 0,0 & 0,2 \\
\hline$:$ & : & $:$ & : & $:$ & $:$ & $:$ & $:$ & : & : & : & $:$ & $:$ \\
\hline 655 & 745,0 & 665,1 & 428,9 & 164,3 & 0,0 & 2,2 & 56,0 & 0,0 & 2,3 & 11,4 & 0,0 & 10,7 \\
\hline Total & $61.354,0$ & $44.471,4$ & $27.618,8$ & $6.083,9$ & 234,7 & $1.354,9$ & $1.271,6$ & 197,5 & $3.310,1$ & $4.268,2$ & 131,5 & 100 \\
\hline
\end{tabular}

(*) Superficie disponible para forestar al año 1998 en cada parcela. Incluye uso actual del suelo agrícola, matorrales.

Representación cartográfica de la proyección del cambio para el período 1998-2002: Con el objeto de facilitar la representación cartográfica de los valores de probabilidad calculados para cada propiedad y UHT (cuadro 5), se agrupó esta información en cuatro categorías: alta probabilidad, media, baja y sin probabilidad de cambio, para el período 1998-2002.

La flexibilidad en el despliegue de la información gráfica dispuesta de esta forma en un modelo vectorial de datos permite modificar la asignación de estas categorías, dependiendo de los objetivos y restricciones específicas de cada estudio y de la experiencia y conocimiento empírico del área.

En la figura 5 se representa cartográficamente la localización de áreas susceptibles de cambio a usos de plantaciones forestales, según el modelo espacial propuesto.

\section{CONCLUSIONES}

El uso de modelos de proyección cartográfica del cambio en el uso del suelo son herramientas relativamente fiables, dada por la potencia y exactitud espacial que le otorga el uso de bases de datos geográficos integrados a un Sistema de Información Geográfico (SIG). El ejemplo presentado en este trabajo, sobre la transformación del paisaje en un área de la VIII Región-Chile, es una alternativa que permite predecir espacialmen- te la localización de áreas vulnerables al cambio y como una herramienta de análisis, no como una solución para los problemas.

Sus principales limitaciones son:

- La imposibilidad actual de georreferenciar información económica como precios, oferta, demanda o rentabilidad del suelo.

- La calidad y el nivel de información socioeconómica no ha tenido un desarrollo paralelo a la información geográfica para ser integrada en el análisis y modelamiento espacial de cambios de uso del suelo.

A la fecha los modelos de predicción espacial de cambios de uso de suelo han sido utilizados principalmente para estudiar el proceso de deforestación en países tropicales $(9,10,11,12,13,14,15)$.

De estos se puede concluir que los principales efectos que producen las variables explicatorias en este tipo de modelos, son:

- La deforestación y aclareo de los bosques declina rápidamente en la medida que disminuye la distancia a los caminos y vías rurales $(13,16)$.

- El proceso de deforestación es más drástico cuando interactúan las variables: distancia a los caminos, áreas con suelos fértiles para la agricultura y condiciones climáticas apropiadas $(9,17)$. 


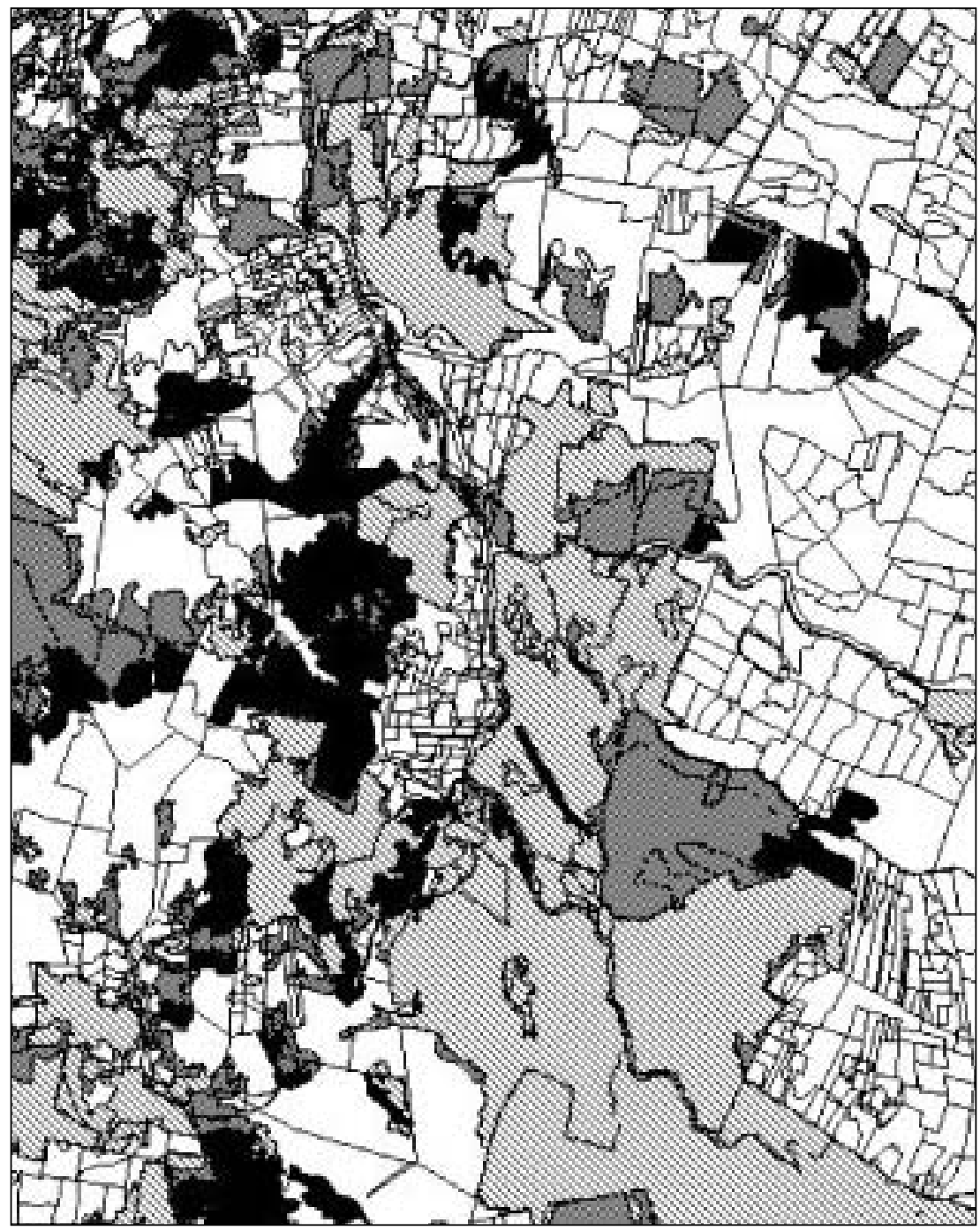

\begin{tabular}{|l|c|}
\hline \multicolumn{1}{|c|}{ LEYENDA } & RANGO DE PROBABILIDAD $\Pi \%$ \\
ALTA & $>15 \%$ \\
MEDIA & $>5 \%$ und $<15 \%$ \\
BAJA & $>0 \%$ und $<5 \%$ \\
PLANTACIONES & $=0 \%$ \\
\hline
\end{tabular}

Figura 5. Distribución espacial de áreas homogéneas y límites de propiedad con diferentes probabilidades de cambio a plantaciones para el período 1999-2002.

Spatial situation of the homgeneadis unids with different change of probability.

- La deforestación declina significativamente al incrementar la distancia a los centros de consumo y mercados. Además, tiene un efecto más significativo que la variable distancia a los caminos rurales $(9,17,18)$.
- Por otra parte, la construcción de caminos disminuye el impacto en los niveles de pobreza (en bosques del sur de México). Pobreza y degradación de los bosques son altamente correlacionados (19). 
- Bosques fragmentados tienen un mayor riesgo de ser deforestados que grandes y compactas extensiones de bosques (16).

\section{BIBLIOGRAFIA}

(1) KAIMOWITZ, D., A. ANGELSEN. Economic Models of Tropical deforestation. A Review Center for International Forestry Research, Malaysia, 1998, 305 p.

(2) LAMBIN, E. Modelling and Monitoring land-cover change processes in tropical regions. Progress in Physical Geography, 1997, vol. 21, No 3, p. 375-393.

(3) TURNER, M., R. GARDNER. Quantitative Methodes in Landscape Ecology, Springer - Verlag, 1990, 536 p.

(4) BAKER, W. L. A review of models of landscape change. Landscape Ecology, 1989, vol. $2 \mathrm{~N}^{\circ} 2$, p. 111-133.

(5) SHUGAR, H., S. SEAGLE. Modeling Forest Landscape and role of disturbance in ecosystems and comunities. In: The Ecology of Natural Disturbance and Patch Dynamics, 1985, p. 353-358.

(6) PARKS, L., R. PETERS., Models of Forested and agricultural Landscape. Integrating Economics. Quantitative Methodes in Landscape Ecology, Springer - Verlag. Capitel, 1990, vol. 12, p. 309-322.

(7) ELSTON, D. A., S.T. BUCLAND. Statistical modelling of regional GIS data. and overview. Ecological Modelling, 1993, vol. 67, p. 81-102.

(8) LAMBIN, E. Modelling Deforestation Processs, A Review. European Commission, Institute for Remote Sensing Apliccations, European Space Agency, 1994, 113 p.

(9) CHOMITZ, K., D. GRAY. Roads, Lands marketz and deforestation, a spatial model of land use en Belize. World Bank Economic Review, 1996, vol. 10, p. 487512.
(10) GASTELLU-ETCHEGORRY, J.P., A.B. SINULINGGA. Designing a GIS for The Study of Forest Evolution in Central Java. Tijdschrift voor Econ. en Soc. Gegrafic, 1988, vol.79, No 2, p. 93-102.

(11) SADER, S., A. JOYCE. Deforestation rates and trends in Costa Rica, 1940-1983. Biotrópica, 1988, vol. 20, p. 11-19.

(12) LUDEKE, A., R.C. MAGGIO, L.M. REID. An analysis of anthropogenic Deforestation Using Logistic Regression and GIS. Journal Of Environmetal Management, 1990, vol. 31, p. 247-259.

(13) LIU, D., L. IVERSON, S. BROWN. Rates and patterns of deforestation in the Plilippines apliccation of geographic information system analysis, Forest Ecology and Management, 1993, vol. 57, p. 1-16.

(14) RINGROSE, S. The use of integrated remotely sensed and GIS data to determine cause of vegetation cover change in southern Botswana. Applied Geography, 1996, vol 16, p. 225-242.

(15) ZERDA, H. Monitoring der Vegetations und Landnutzungsveränderungen durch Brandung und Übernutzung im Trocken- Chaco Argentiniens mit Satellitenfernerkundung un GIS. Göttingen, Cuvillier Verlag, 1998, 175 p.

(16) MAMINGI, N., K.M. CHOMITZ, D.A. GRA., E.F. LAMBIN. Spatial patterns of deforestation in Cameroon and Zeire. Working Paper 8 Research Project on Social and Environmental Consequences of Growht-oriented Policies. Policy Research Department World Bank, Washington, 1996, $35 \mathrm{p}$.

(17) MERTENS, B., E. LAMBIN. Spatial Modelling of deforestation in southern Cameroon. Applied Geography, 1997, vol. $17, \mathrm{~N}^{\circ} 2$, p. $143-162$.

(18) NELSON, G., D. HELLERSTEIN. Do Roads Cause deforestation Using Satellite images in econometric analysis of land use. American Journal of Agricultural Economics, 1997, vol. 79, p. 80-88.

(19) DEININGER, K., B. MINTEN. Determinants of forest cover and the economics of protection. An application to Mexico. World Bank Washington. D.C., 1997. 